\title{
Enrichment of Microbes Potentially Degrading Polyethylene Using a Microcosm Approach
}

\section{Ana Maria Barral and Ben Stenson National University, San Diegu, CA}

National University

11255 North Torrey Pines Road, La Jolla, CA 92037

Contact Email: abarral@nu.edu

Manuscript received 12 May 2020; accepted 11 June 2020.

$84 \mid$ Fine Focus 


\section{Abstract}

Plastic pollution is a worldwide phenomenon with concerning effects on the biosphere and particularly on the marine environment. Biodegradation is considered an environmentally friendly alternative to combat the increasing quantities of plastic pollutants where different microbial sources are tested for plastic degradation potential. In this project, a microcosm approach was used as an enrichment method for marine microbes degrading polyethylene. Pieces of low-density polyethylene (LDPE) and highdensity polyethylene (HDPE) previously deployed in ocean water have been explored as a source of microbial biomass. This source plastic was added to a synthetic medium containing sterilized pieces of LDPE and HDPE as the sole carbon source and were incubated for extended periods (32-86 days) in the laboratory to promote growth of microbes that can degrade plastic. Biodegradation of polyethylene was confirmed by dry weight measurements and Fourier Transform Infra-Red (FTIR) spectroscopy. For both LDPE and HDPE a significant reduction in dry weight was observed. FTIR analysis showed peaks suggesting oxidative changes in polyethylene's chemical composition. In summary, the microcosm approach can be considered a viable approach for enrichment of plastic-degrading marine microbial populations. 


\section{Introduction}

Few topics span so many disciplines and touch the public interest as much as the issue of plastic pollution. Plastic is an essential element of our everyday lives, and its introduction in multiple spheres of human activity brought economically and scientifically sensible solutions and innovations. Plastic can be defined as long-chain synthetic polymers created through polymerization of monomers obtained from oil or gas with chemical additives (1). Plastic material is used to package almost every commodity, and it is estimated that one-third of plastic manufactured is used in disposable one-time use products (2). Due to its non-degradable nature, plastic has been accumulating in the environment at exponentially increasing rates since mass production began in the 1940 s $(1,3)$. Some of the plastic accumulating in the environment inevitably makes its way into the ocean environment. Rain, wind, tsunamis, streams, and other phenomena carry plastics into the ocean, where they fragment into microscopic pieces. Additionally, microplastics may enter the marine environment through cleaning scrubbers, abrasive beads for cleaning boats, and deterioration of larger pieces of plastic (4). Plastic litter and contaminants can be found on remote beaches throughout the world constituting not only an eyesore, but also a hazard for the environment. Dangers of plastic include harm to animals (5), ability to transport contaminants around the ocean (6), as well as release of toxic chemicals upon degradation (4, $7,8)$.

Diverse abiotic and biotic processes can degrade plastic in marine environments (9-11). Degradation can be defined as a process that leads to a decline of polymerlike properties through multiple steps. Weathering is the physical deterioration of plastic, which occurs in the ocean through wind and waves. Photodegradation is the leading cause for degradation of plastic, as UV-B radiation from sunlight breaks the $\mathrm{C}-\mathrm{H}$ bonds on the polymer backbone, creating a carbon free radical that most often reacts with oxygen creating a peroxy radical. Olefins, aldehydes, and ketones are the most common products, but C-C crosslinking can also occur (11). Photo-initiated oxidative degradation is the rate determining step of degradation in the marine environment.

Biotic degradation is not as well documented as abiotic processes $(12,13)$ and it is almost always preceded by abiotic degradation (14). Microbes can excrete extracellular enzymes, which break down and utilize the carbon backbone of most plastics. In the marine environment, conditions are not optimal, so both abiotic and biotic degradation occur slowly on a scale of decades. Potential microbial candidates for biodegradation of plastic have been previously described, including a Penicillum species from a landfill (15), Pseudomonas (16, 17) Bacillus (18) and Rhodococcus (19) among others. Recent reviews list a number of marine microbial groups with described or potential plastic degradation ability (20-22).

Polyethylene (PE) is the most common plastic and can be found in two varieties: high-density PE (HDPE) and low-density PE (LDPE). PE represents 64\% of all plastic produced and it is the most common floating plastic as well as the most common plastic found on beaches (23). Due to its light weight and hydrophobicity, PE floats in ocean water. Its backbone is composed solely of carbon atoms, which can be the target of microbial activity originating at any terminal methyl group.

Previous studies have described the "plastisphere," a unique microbial ecosystem living on and in floating plastic, containing organisms potentially able to use plastic polymers as a food source $(12,13,24,25)$. An ongoing study exploring the bacterial populations 
attaching to floating plastic in coastal waters (26) has produced plastic with a rich biomass. We hypothesized that this biomass could be a suitable source for the identification of potentially plastic-degrading microbes.

The microcosm approach, first described in the 19th century (27) has been a debated model to recreate complex ecological systems in a laboratory environment (28-30). A number of authors have used this approach to isolate and identify microbes with the ability to degrade plastic from diverse complex sources (31-33) In this study, we tested a microcosm approach to enrich for marine microbes with the ability to degrade PE. We used PE previously exposed to ocean water as microbial source, and UV-irradiated $\mathrm{PE}$ as the sole carbon source in the growth medium. Plastic degradation was tested using chemical methods such as Fourier Transform Infrared Spectroscopy (FTIR) and dry weight measurements. Our results indicate modest degradation by decrease in weight and observed oxidative changes.

\section{Materials and Methods}

\section{Microcosm}

Figure 1 shows the overall setup of the microcosm experiment to select plastic degrading microbes. For microbial source, PE that had been previously exposed to ocean water (either in the field or in a laboratory setting, see section below) was added to a synthetic medium containing no carbon. As a carbon source, sterilized PE was used. Sterile mason jars were used to contain the medium.

\section{Microbial Source}

As source of microbial biomass, PE samples previously incubated in ocean water were employed. These samples are part of a project studying microbial colonization in coastal waters (26). Briefly, plastic squares about $5 \mathrm{~cm} \times 5 \mathrm{~cm}$ were sterilized and placed in sampling cages, which were either attached to the Scripps Oceanography pier in San Diego, CA (GPS coordinates $32^{\circ} 52^{\prime} 00.8^{\prime \prime} \mathrm{N} 117^{\circ} 15^{\prime} 28.1$ 'W, approximately $5 \mathrm{~m}$ under the water surface), or placed in $5 \mathrm{~L}$ carboys containing ocean water in the laboratory incubator set at $16 \mathrm{oC}$. Microbial biomass was collected on three different occasions, after 33 days (pier), 153 days (lab), and 70 days (lab) incubations. Collection times were variable due to both weather conditions and a related educational field trip experience (34). Samples were collected using gloves and sterilized instruments, and placed in sterile pouches. The collected plastic samples with the attached biomass were used as microbial sources for three sets of microcosm experiments. A $3 \mathrm{~cm} \mathrm{x}$ $3 \mathrm{~cm}$ piece of PE (either HDPE or LDPE), cut with sterile scissors using aseptic technique was added to the microcosms. Control cultures with no microbial source were set up in parallel for the first microcosm experiment.

\section{Source of PE}

Sources of high-density PE (HDPE) and low-density PE (LDPE) were clean produce and grocery bags, respectively. The same batches of bags collected from Sprouts and Amazon packaging were used for all experiments. The chemical nature of each polymer was confirmed through FTIR (see section below).

\section{Pretreatment of PE}




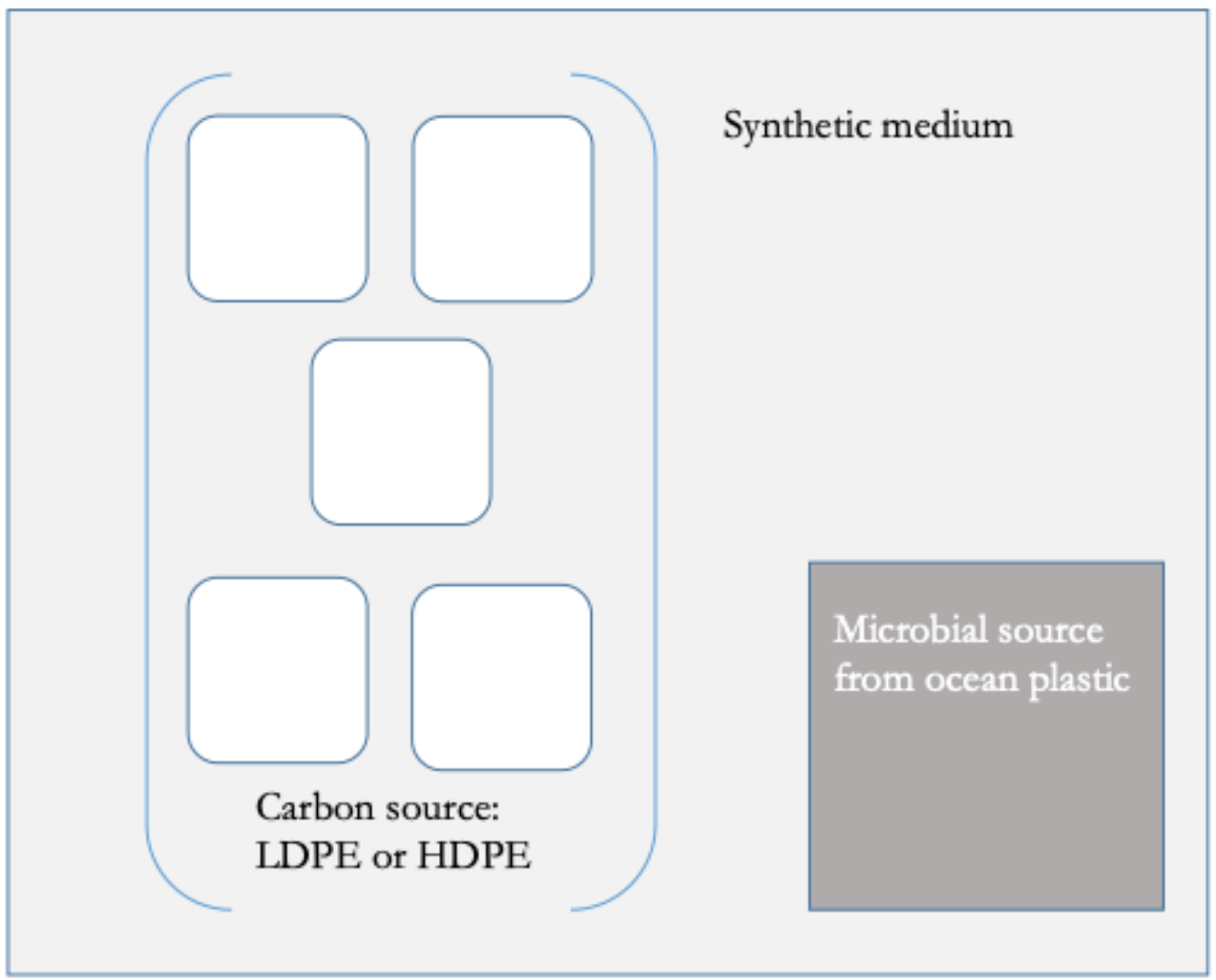

Figure 1. Microcosm setup. PE pieces previously incubated in the ocean were used as source of microbes. The synthetic medium imitated ocean salinity but contained no carbon source. Carbon was provided as sterilized PE fragments ( 5 per culture).

88 Fine Focus 
Prior to placement in medium, the PE used as carbon source was sterilized through serial washes with shaking (40 min in SDS 2\%, $15 \mathrm{~min}$ in deionized water, $30 \mathrm{~min}$ in $70 \%$ ethanol), using a method adapted from Das \& Kumar (18). After the last wash, the PE pieces were handled using aseptic technique, stored in sterilized pouches, and left to dry overnight at $60^{\circ} \mathrm{C}$. To simulate photo-initiated degradation (naturally occurring in the marine environment by solar radiation), PE samples were incubated under a UVP UV emitter (Model TM-10E, Upland, California, USA). Plastic was cut into $1 \mathrm{~cm} \times 1 \mathrm{~cm}$ squares using aseptic technique and exposed to UV light at $253.7 \mathrm{~nm}$ for 48 total hours. Five squares per sample were added to each microcosm experiment.

\section{Culture Conditions}

For the microcosm experiment, cultures were kept in a previously described synthetic medium $(14)$, at $16^{\circ} \mathrm{C}$ in the dark. The medium mimicked ocean salinity while containing no carbon sources other than the added plastic. It contained: $1 \mathrm{~g} \mathrm{NH}_{4} \mathrm{NO}_{3}, 1 \mathrm{~g} \mathrm{~K} \mathrm{HPO}_{4}$, $0.15 \mathrm{~g} \mathrm{KCL}, 0.2 \mathrm{~g} \mathrm{MgSO}_{4}, 0.1 \mathrm{~g} \mathrm{CaCl}_{2}, 0.1 \mathrm{~g}$ yeast extract, $1 \mathrm{mg} \mathrm{MnSO}_{4}, 1 \mathrm{mg} \mathrm{FeSO}$, and $1 \mathrm{mg} \mathrm{ZnSO}_{4}$ per 1L distilled water. Cultures were set up in $250 \mathrm{~mL}$ sterile mason jars. Hundred $\mathrm{mL}$ of the medium were supplemented with 5 pieces of $1 \mathrm{~cm} \times 1 \mathrm{~cm}$ PE squares and one piece of previously incubated PE as microbial source. Paraffin oil, a non-ionic surfactant, was added at a final concentration of $0.05 \% \mathrm{v} / \mathrm{v}$ to the second and third experimental sets to enhance colonization (17). Three sets of microcosm experiments were completed for a length of 32,70 , and 86 days.

\section{Determination of Dry Weight}

Weight of the supplemented PE was recorded after the plastic had been sterilized and left to dry overnight at $60^{\circ} \mathrm{C}$, but before it was transferred to the synthetic medium ("before"). After the incubation time concluded, plastic was treated with the same sterilization and drying process and weighed again (“after") on a Mettler Toledo scale (Model XS603S, Switzerland). The scale was previously calibrated according to the manufacturer's protocol and had a sensitivity of $\pm 0.01 \mathrm{mg}$. Dry weight measurements were compared using a t-test.

\section{FTIR Analysis}

FTIR studies were performed on the individual PE pieces at the end of the incubation after the dry weight was recorded. As controls PE fragments sterilized and UV-treated but not incubated were used. FTIR was performed on a Nicolet iZ10 Spectrometer and spectra analyzed using the Omnic Anywhere Cloud Computing system (Thermo Scientific, Waltham, $\mathrm{MD})$. The double bond index (DBI) was calculated based on the relative intensity of the double bond band at $1,650 \mathrm{~cm}-1$ to that of the methylene scissoring band at $1,460 \mathrm{~cm}-1$ (35). The DBI of the control and the experimental samples was compared using a one-way ANOVA. For statistical analyses the GraphPad Prism version 8.0.0 for Mac was used (GraphPad Software, San Diego, CA).

\section{Results}

\section{Microcosm experiments}

Table 1 shows the details of the microbial sources and incubation times for the three microcosm experiments. Pier and laboratory incubations had been set up in parallel to guarantee samples for an educational project, using the same sampling system and as similar conditions as possible. Laboratory experiments used ocean water from the pier location. Water temperatures at the Scripps pier were averaged based on the daily readings from the incubation 
Table 1. Microcosm experiments and the corresponding microbial sources.

\begin{tabular}{|l|l|l|l|l|}
\hline $\begin{array}{l}\text { Microcosm } \\
\text { set }\end{array}$ & $\begin{array}{l}\text { Location } \\
\text { of original } \\
\text { incubation }\end{array}$ & $\begin{array}{l}\text { Length of original } \\
\text { incubation (days) }\end{array}$ & $\begin{array}{l}\text { Water temperature } \\
\left({ }^{\circ} \mathbf{C}\right)\end{array}$ & $\begin{array}{l}\text { Length of microcosm } \\
\text { experiment (days) }\end{array}$ \\
\hline $\mathbf{1}^{*}$ & Pier & 33 & 15.7 (average)** & 32 \\
\hline $\mathbf{2}$ & Laboratory & 153 & 16 & 70 \\
\hline $\mathbf{3}$ & Laboratory & 70 & 16 & 86 \\
\hline
\end{tabular}

*This set also included a negative control (no microbial source added).

**Data provided by the Shore Stations Program, with current funding provided by the California Department of Parks and Recreation, Division of Boating and Waterways, Award\# C1670003. Data are collected by staff aquarists and volunteers with the Birch Aquarium at Scripps. 


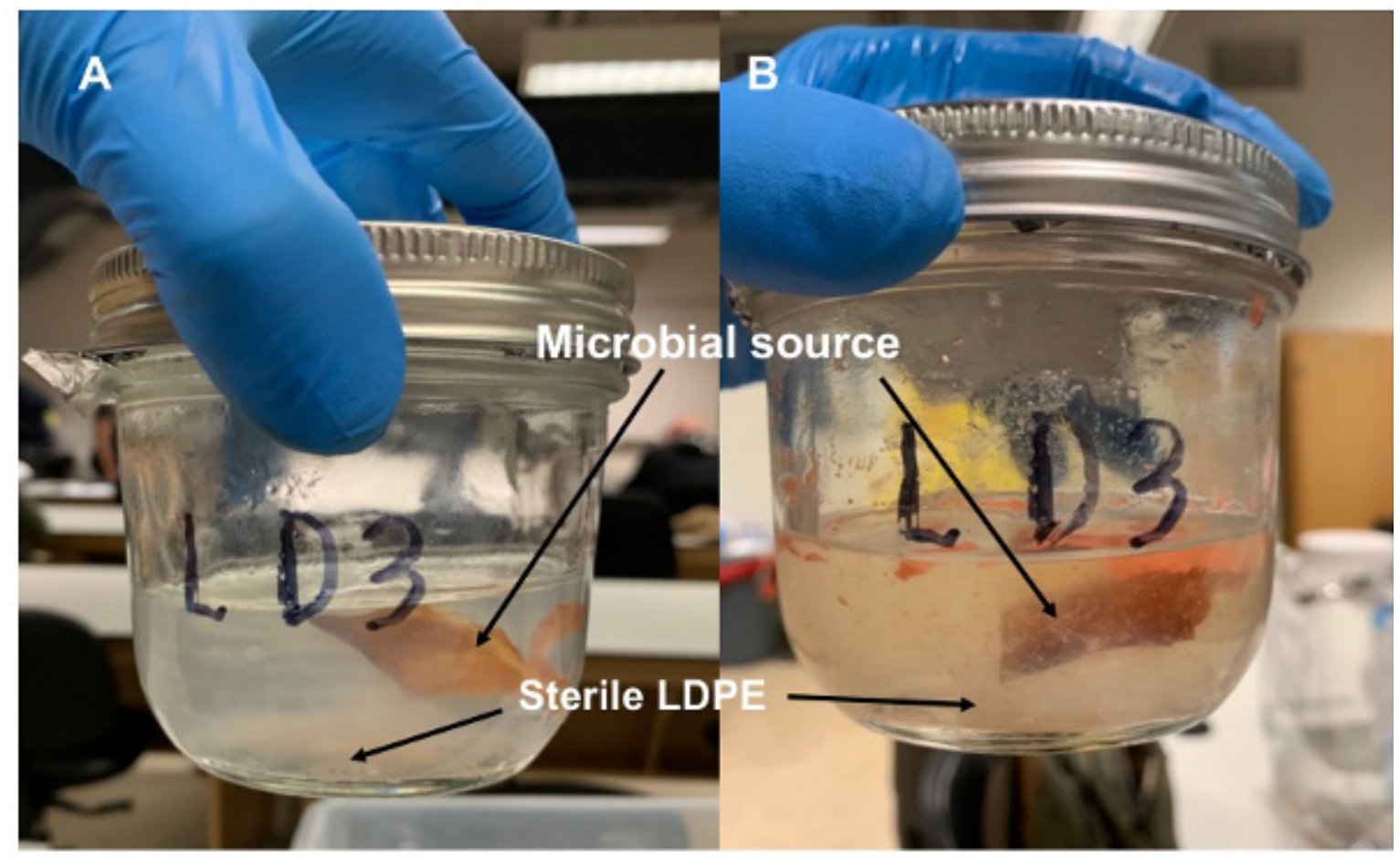

Figure 2. A: a LDPE microcosm at the beginning of incubation. B: the same microcosm at the end of a 70-day incubation. 
period, while the temperature was set at 16 oC for the laboratory incubations.

Figure 2 shows a LDPE sample from the set \#2 microcosm experiment at day 0 and day 70 , respectively. Increased turbidity can be observed in the medium. Overall, microcosm samples consistently showed microbial growth as evidenced by increased turbidity. Uneven loss of volume due to evaporation was observed, preventing measurement of turbidity as a reflection of growth.

\section{Dry weight}

Dry weight of the plastic specimens was measured before and after incubation. Due to the extensive manipulation required for the sterilization and drying of the PE samples, some HDPE samples were lost during the process. The negative controls (PE incubated in the microcosm without a microbial source) from set \#1 did not show reduction in weight.

Weight loss was recorded in $69 \%$ of samples while no change was recorded in $31 \%$ of samples. There was no clear relationship between weight loss and length of incubation tested or source of microbial culture. Figure 3 shows the dry weight change in all HDPE and LDPE samples tested before and after the incubation. The average weight for LDPE samples decreased from $11.4 \mathrm{mg}(\mathrm{SD}=1.35)$ to $10.1 \mathrm{mg}(\mathrm{SD}=1.37)$. Similarly, HDPE weight decreased from $5.5 \mathrm{mg}(\mathrm{SD}=2.9)$ to $4.5 \mathrm{mg}(\mathrm{SD}=2.17)$. The decrease of dry weight was statistically significant for both $\operatorname{LDPE}(\mathrm{p}=.0133$, $t=3.074, d f=9)$ and HDPE $(p=.0409, t=2.739, d f=5)$.

\section{FTIR}

When comparing the FTIR spectra of control (pretreated but not included in the microcosm) PE with the microcosm LDPE and HDPE samples, peaks indicative of oxidative processes could be observed. Figure 3 shows the FTIR spectra corresponding to representative LDPE and HDPE samples after 70 days. Compared to the control, both LDPE and HDPE show additional peaks corresponding to the range of alkynes $\left(2,200-2,300 \mathrm{~cm}^{-1}\right)$ as well as a robust peak corresponding to double bonds such as the carbonyl group $\left(1,640-1,680 \mathrm{~cm}^{-1}\right)$.

Figure 5. shows the double-bond index (DBI) calculated for each microcosm set. There is no significant difference between the control and the day 30 samples for either LDPE and HDPE. While there seems to be an overall increase in the DBI for both LDPE and HDPE by day 70, only in HDPE was the increase significant from $\mathrm{M}=0.200(\mathrm{SD}=.0956)$ to $\mathrm{M}=0.403(\mathrm{SD}=.07319)(\mathrm{p}=.0063)$. The day 86 samples were not significantly different from the control.

\section{Discussion}

The term "great plate count anomaly" was coined by Staley and Konopka in 1985, which described the discrepancy between total microbial counts from a natural environment and colonies isolated by traditional microbiology methods (36). It is estimated that the latter can only recover $1 \%$ or less of the bacterial diversity in most environmental samples (37). Therefore it has been a continuing challenge for microbial ecologists to describe complex microbial populations and the key interactions between their components. Culture-independent methodologies, based on DNA analysis using metagenomic approaches are often used to characterize complex populations (38). Such analyses have helped the identification of microbes whose genomes contain 


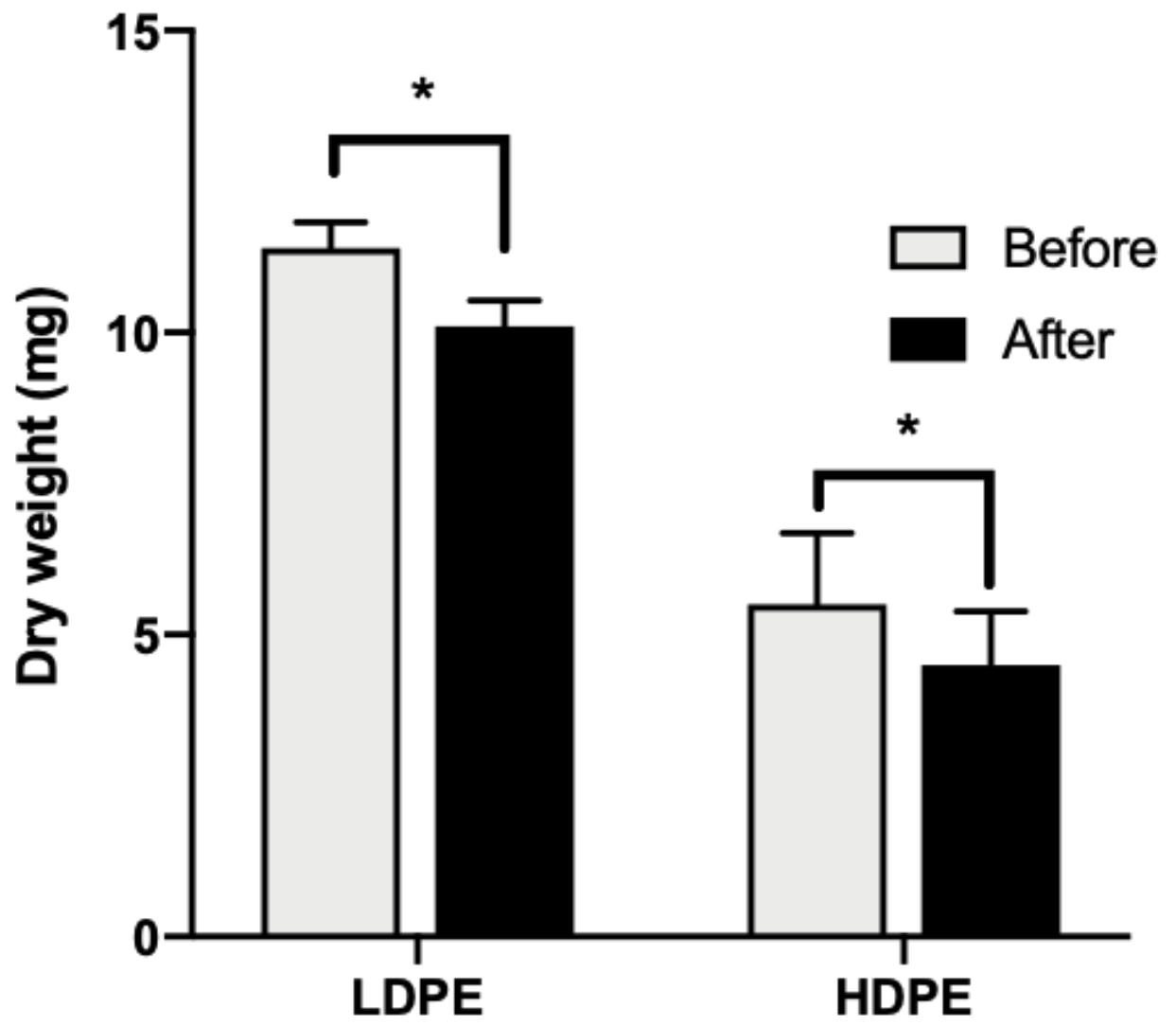

Figure 3. Average dry weight significantly decreased for both LDPE ( $M=11.4 \mathrm{mg}, \mathrm{SD}=1.35$ to $\mathrm{M}=10.1 \mathrm{mg}, \mathrm{SD}=1.37)$ and $\mathrm{HDPE} \mathrm{M}=5.5 \mathrm{mg}, \mathrm{SD}=2.89$ to $\mathrm{M}=4.5 \mathrm{mg}, \mathrm{SD}=2.17$ ) after the microcosm incubation. As controls PE fragments sterilized and UV-treated but not incubated were used. The * indicates significant differences of $\mathrm{p}=.0133$ and $\mathrm{p}=.0409$ for LDPE and HDPE, respectively. 


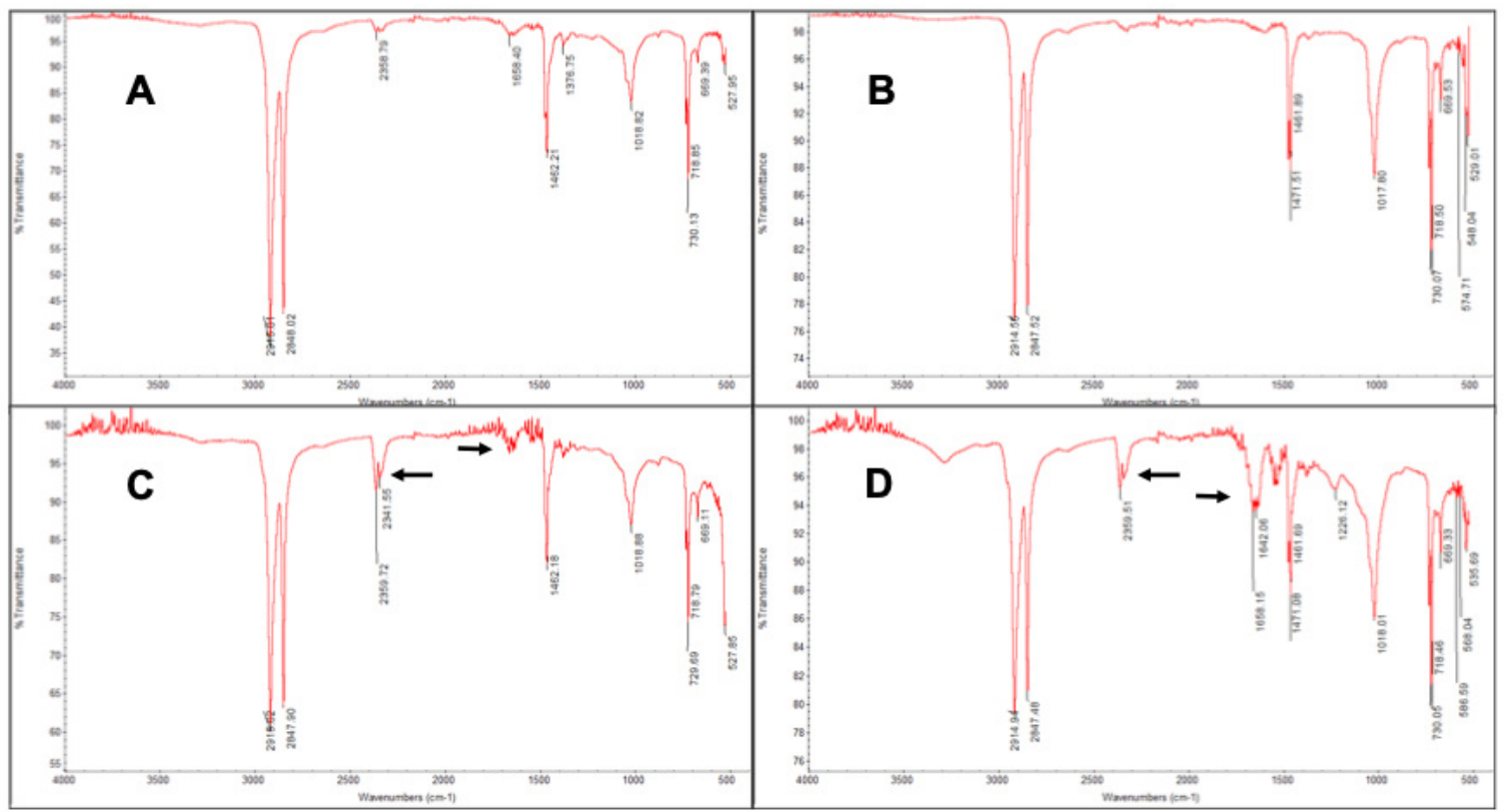

Figure 4. Representative FTIR profiles of LDPE (A-before, C-after) and HDPE (B-before, D-after). Distinct peaks corresponding to alkynes and double bonds are highlighted with arrows. 

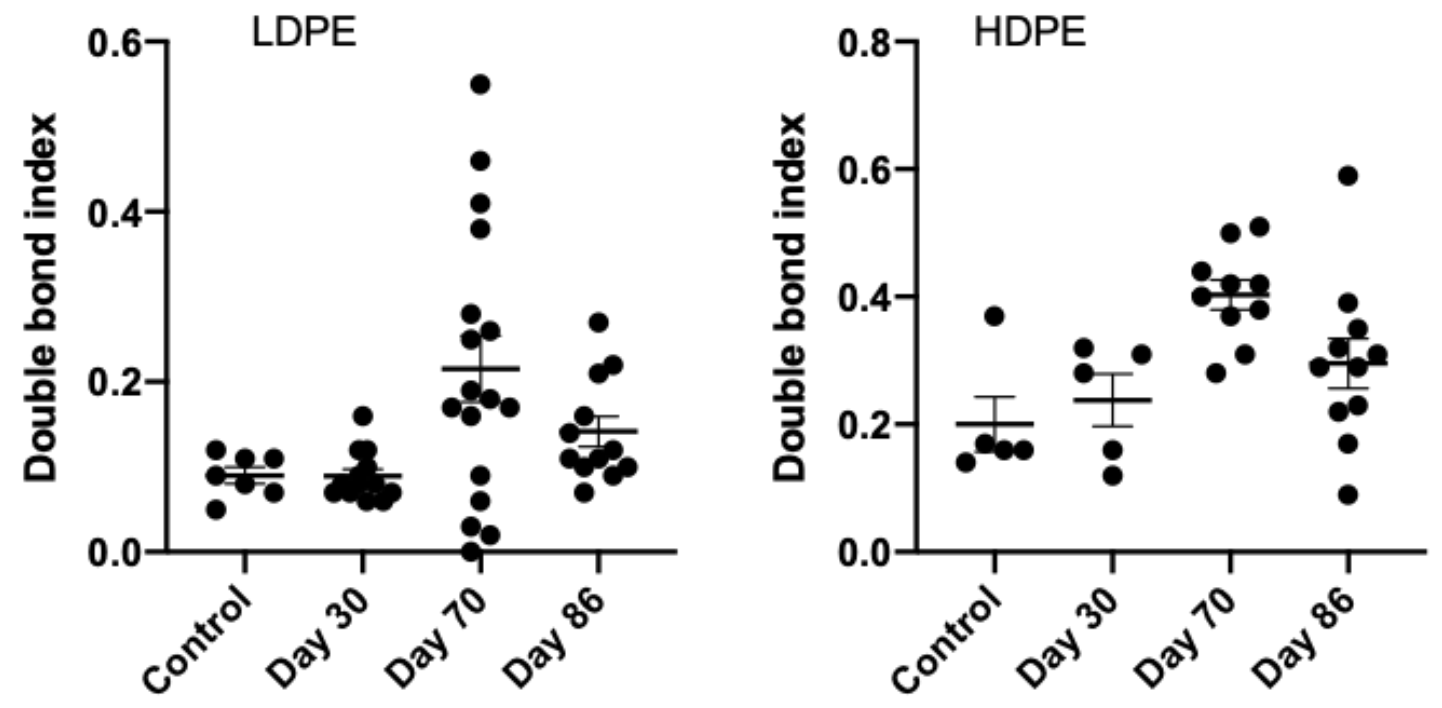

Figure 5. Double bond index values calculated from the samples of the three microcosm experiments using FTIR. 
genes for enzymes that may be involved in plastic degradation (12). However, interest for the isolation and characterization of microbes using culturedependent approaches remains, particularly for marine organisms (39).

A microcosm is a miniature, controlled environment (40) that can act as proxy of complex populations (30, 41). In this study, it served as a "low-tech" approach to select microbial entities with the potential to degrade plastic from a complex biomass. Results showed presence of microbial populations capable of degrading LDPE and HDPE as assessed by decrease in dry weight and chemical changes detected with FTIR.

Previous studies have shown chemical signs of degradation in $\mathrm{PE}$ and polypropylene samples deployed in coastal waters $(26,42-44)$. The microcosm approach of this article was developed to promote the potential biodegradation process in a controlled environment while limiting abiotic processes such as weathering and photodegradation. The setup was conducive to microbial growth, which could be observed as increased turbidity, especially after the addition of paraffin oil to the synthetic medium. Paraffin oil has been described to increase microbial attachment and formation of a biofilm, which ultimately results in enhanced degradation (17). Due to evaporation of culture media in some samples, measurement of absorbance of the culture was deemed not reliable, an aspect that should be improved in future experiments.

Field experiments are notorious for the influence of factors outside the control of researchers. The first set of the microcosm experiments had a microbial source resulting from an incubation in the ocean for 33 days, and itself had a duration of 32 days. Based on the literature and our data showing minimal changes in the FTIR spectra, a decision was made to test microbial sources resulting from laboratory incubations that could be kept for longer times. These incubations were completed at controlled temperatures in large carboys containing ocean water. Sets \#2 and \#3 of the microcosm experiments, using the laboratory microbial sources, exhibited more growth and stronger signs of degradation according to FTIR, which seems related to the length of the original incubation. It has been described previously that plastic biodegradation is an extremely slow process (9), as also evidenced by our results. While clear FTIR peaks indicative of oxidation were observed after more than 2 months incubation, LDPE and HDPE lost only an average of $11 \%$ and $18 \%$ of weight, respectively.

FTIR spectroscopy is a commonly used analytical tool to detect changes in chemical composition. Formation of new functional groups as well as the disappearance of others are indicative of significant chemical changes (18). Our results are in agreement with previous authors' findings that show signs of PE oxidation as a result of biodegradation. Interestingly, we did not observe a defined carbonyl stretch peak at $1712 \mathrm{~cm}^{-1}$, a typical finding in UV-treated PE, which is often used as a measure of photodegradation (45). However, the presence of double bonds increased, as shown by both a larger peak in the range of $1640-1680 \mathrm{~cm}^{-1}$ and the concurrent changes in double bond index, particularly for HDPE at day 70. This could be explained by microbial activity attacking the carbonyl groups generated by abiotic factors, resulting in unsaturated chains (35).

The fact that only HDPE from the second (70-day) set showed a significant difference compared to the control, while the 86-day third set HDPE did not, is intriguing. However, the second set's microbial source originated from a 153 day incubation, more 
than double the length of the third set experiment's source (70 days). Enrichment of PE-degrading microbes may have already taken place in the source material of the second set. Previous FTIR analyses of ocean-incubated samples have shown that signs of degradation appear first for HDPE and later for LDPE (manuscript in preparation). If this is due to chemical differences in HDPE and LDPE, or to different physical characteristics is not yet known.

The FTIR results highlight some of the limitations of this study, which are standardization and repeatability. As a side project to a field-based educational project that is highly dependent on weather conditions and logistical issues, these initial samples had microbial sources with variable incubation conditions. However, overall the results seem to suggest that 1 ) laboratory samples to generate microbial sources are as effective as field samples, and 2) length of the original incubation is a paramount factor.

Metagenomic characterization of the biomass and surrounding water is ongoing. Preliminary results indicate an extensive overlap between plastic and water, which shifts over time; as well as a high prevalence of unknown taxa (Dr. R. Simmons, personal communication). Another ongoing study uses culture-dependent methods by swabbing media plates with the biomass to characterize the resulting colonies via 16S PCR (26).

Future experiments will continue the microcosm approach to enrich in plastic-degrading bacteria using longer incubation times to achieve even higher degradation rates. To isolate and characterize the microbes responsible for degrading PE, standard microbiology dilution techniques combined with selective media will be used, similar to the approach used to isolate a PET-degrading bacterium (46). We plan to use metagenomic sequencing of the original microbial sources and the microcosm cultures to characterize changes in populations. In addition, whole genome sequencing of the population may provide clues to the enzymes involved in the degradation pathway.

\section{Acknowledgments}

The authors would like to thank Dr. Jeff Bowman at Scripps Institution of Oceanography (UCSD), Dr. Emilia DeForce of Thermo Fisher Scientific, and Dr. Rachel Simmons of National University for making this project possible. We are grateful to Ms. Juliann Downing of National University for the invaluable help with the FTIR analysis. This project was supported by the National Science Foundation (NSF) award \#1832545. Any opinions, findings, and conclusions or recommendations expressed in this material are those of the author(s) and do not necessarily reflect the views of the National Science Foundation. 


\section{References}

1. Thompson RC, Swan SH, Moore CJ, vom Saal FS. 2009. Our plastic age. Philos Trans R Soc Lond B Biol Sci 364:1973-6.

2. Geyer R, Jambeck JR, Law KL. 2017. Production, use, and fate of all plastics ever made. Sci Adv. 3: e1700782

3. Ostle C, Thompson RC, Broughton D, Gregory L, Wootton M, Johns DG. 2019. The rise in ocean plastics evidenced from a 60-year time series. Nat Commun 10:1622.

4. Teuten EL, Saquing JM, Knappe DRU, Barlaz MA, Jonsson S, Björn A, Rowland SJ, Thompson RC, Galloway TS, Yamashita R, Ochi D, Watanuki Y, Moore C, Viet PH, Tana TS, Prudente M, Boonyatumanond R, Zakaria MP, Akkhavong K, Ogata Y, Hirai H, Iwasa S, Mizukawa K, Hagino Y, Imamura A, Saha M, Takada H. 2009. Transport and release of chemicals from plastics to the environment and to wildlife. Philos Trans R Soc Lond B Biol Sci 364:2027-45.

5. Derraik JGB. 2002. The pollution of the marine environment by plastic debris: A review. Mar Pollut Bull 44:842-852.

6. Law KL, Thompson RC. 2014. Oceans. Microplastics in the seas. Science 345:144-5.

7. Moore CJ. 2008. Synthetic polymers in the marine environment: A rapidly increasing, long-term threat. Environ Res 108:131-139.

8. Van A, Rochman CM, Flores EM, Hill KL, Vargas E, Vargas SA, Hoh E. 2012. Persistent organic pollutants in plastic marine debris found on beaches in San Diego, California. Chemosphere 86:258-63.

9. Shah AA, Hasan F, Hameed A, Ahmed S. 2008. Biological degradation of plastics: A comprehensive review. Biotechnol Adv 26:246-265.

10. O’Brine T, Thompson RC. 2010. Degradation of plastic carrier bags in the marine environment. Mar Pollut Bull 60:2279-2283.

11. Gewert B, Plassmann MM, MacLeod M. 2015. Pathways for degradation of plastic polymers floating in the marine environment. Environ Sci Process Impacts 17:1513-1521.

12. Quero GM, Luna GM. 2017. Surfing and dining on the "plastisphere": Microbial life on plastic marine debris. Adv Oceanogr Limnol 8. https://doi.org/10.4081/aiol.2017.7211

13. Zettler ER, Mincer TJ, Amaral-Zettler LA. 2013. Life in the "plastisphere": Microbial communities on plastic marine debris. Environ Sci Technol 47:7137-7146.

14. Hadad D, Geresh S, Sivan A. 2005. Biodegradation of polyethylene by the thermophilic bacterium Brevibacillus borstelensis. J Appl Microbiol 98:1093-1100.

98 | Fine Focus 
15. Sowmya H V., Ramalingappa, Krishnappa M, Thippeswamy B. 2015. Degradation of polyethylene by Penicillium simplicissimum isolated from local dumpsite of Shivamogga district. Environ Dev Sustain 17:731-745.

16. Tribedi P, Sarkar S, Mukherjee K, Sil AK. 2012. Isolation of a novel Pseudomonas sp from soil that can efficiently degrade polyethylene succinate. Environ Sci Pollut Res 19:2115-2124.

17. Tribedi P, Sil AK. 2013. Low-density polyethylene degradation by Pseudomonas sp. AKS2 biofilm. Environ Sci Pollut Res 20:4146-4153.

18. Das MP, Kumar S. 2015. An approach to low-density polyethylene biodegradation by Bacillus amyloliquefaciens. 3 Biotech 5:81-86.

19. Orr IG, Hadar Y, Sivan A. 2004. Colonization, biofilm formation and biodegradation of polyethylene by a strain of Rhodococcus ruber. Appl Microbiol Biotechnol 65:97-104.

20. Roager L, Sonnenschein EC. 2019. Bacterial Candidates for Colonization and Degradation of Marine Plastic Debris. Environ Sci Technol 53:11636-11643.

21. Jacquin J, Cheng J, Odobel C, Pandin C, Conan P, Pujo-Pay M, Barbe V, Meistertzheim AL, Ghiglione JF. 2019. Microbial ecotoxicology of marine plastic debris: A review on colonization and biodegradation by the "plastisphere." Front Microbiol.10. https://doi.org/10.3389/fmicb.2019.00865

22. Ghosh S, Qureshi A, Purohit HJ. 2019. Microbial degradation of plastics: Biofilms and degradation pathways. In: Contaminants in Agriculture and Environment: Health Risks and Remediation.Vol 1. Agro Environ Media. DOI: 10.26832/AESA-2019-CAE-0153-014

23. Sivan A. 2011. New perspectives in plastic biodegradation. Curr Opin Biotechnol 22:422-6.

24. Law KL, Morét-Ferguson SE, Goodwin DS, Zettler ER, Deforce E, Kukulka T, Proskurowski G. 2014. Distribution of surface plastic debris in the eastern pacific ocean from an 11-year data set. Environ Sci Technol 48:4732-4738.

25. Amaral-Zettler L, Zettler E, Slikas B, Boyd G, Melvin D, Morrall C, Proskurowski G, Mincer T. 2015. The biogeography of the Plastisphere: implications for policy. Front Ecol Env 13:541-546.

26. Leask A, Barral AM, Simmons RE. 2020. Distinct Bacterial Populations Colonizing Plastic Debris in Coastal Waters of Southern California. FASEB J.34.1-1. https://doi.org/10.1096/fasebj.2020.34. s1.04360

27. Beyers RJ. 1964. The Microcosm Approach to Ecosystem Biology. Am Biol Teach 26:491-498.

28. Benton TG, Solan M, Travis JMJ, Sait SM. 2007. Microcosm experiments can inform global ecological problems. Trends Ecol Evol.22. 516-21. 
29. Srivastava DS, Kolasa J, Bengtsson J, Gonzalez A, Lawler SP, Miller TE, Munguia P, Romanuk T, Schneider DC, Trzcinski MK. 2004. Are natural microcosms useful model systems for ecology? Trends Ecol Evol 19:379-384.

30. Drake JM, Kramer AM. 2012. Mechanistic analogy: How microcosms explain nature. Theor Ecol 5:433444.

31. E Syranidou KKFA. 2017. Biodegradation of weathered polystyrene films in seawater microcosms. Sci Rep 7:17991.

32. Harrison JP, Schratzberger M, Sapp M, Osborn A. 2014. Rapid bacterial colonization of low-density polyethylene microplastics in coastal sediment microcosms. BMC Microbiol 14:232.

33. Delacuvellerie A, Cyriaque V, Gobert S, Benali S, Wattiez R. 2019. The plastisphere in marine ecosystem hosts potential specific microbial degraders including Alcanivorax borkumensis as a key player for the low-density polyethylene degradation. J Hazard Mater 380.120899. doi:10.1016/j. jhazmat.2019.120899

34. Barral AM, Simmons RE, Boman-Davis M, Bowman JS. 2020. CUREing Ocean Plastic Microbes: Positive Experience of Engaging Non-traditional Undergraduate Students in a Hispanic-Serving Institution. FASEB J.34. 1-1. https://doi.org/10.1096/fasebj.2020.34.s1.04302

35. Esmaeili A, Pourbabaee AA, Alikhani HA, Shabani F, Esmaeili E. 2013. Biodegradation of Low-Density Polyethylene (LDPE) by Mixed Culture of Lysinibacillus xylanilyticus and Aspergillus niger in Soil. PLoS One.8. e71720. https://doi.org/10.1371/journal.pone.0071720

36. Staley JT, Konopka A. 1985. Measurement of in Situ Activities of Nonphotosynthetic Microorganisms in Aquatic and Terrestrial Habitats. Annu Rev Microbiol 39:321-346.

37. Amann RI, Ludwig W, Schleifer KH. 1995. Phylogenetic identification and in situ detection of individual microbial cells without cultivation. Microbiol Rev 59:143-69.

38. Riesenfeld CS, Schloss PD, Handelsman J. 2004. Metagenomics: Genomic Analysis of Microbial Communities. Annu Rev Genet. 38:525-552

39. Connon SA, Giovannoni SJ. 2002. High-throughput methods for culturing microorganisms in very-lownutrient media yield diverse new marine isolates. Appl Environ Microbiol 68:3878-3885.

40. Lasserre P, Tournié T. 1984. Use of microcalorimetry for the characterization of marine metabolic activity at the water-sediment interface. J Exp Mar Bio Ecol. 74:123-139.

41. Osterholz H, Niggemann J, Giebel H-A, Simon M, Dittmar T. 2015. Inefficient microbial production of refractory dissolved organic matter in the ocean. Nat Commun 6:7422.

100 | Fine Focus 
42. Oberbeckmann S, Labrenz M. 2020. Marine Microbial Assemblages on Microplastics: Diversity, Adaptation, and Role in Degradation. Ann Rev Mar Sci 12:209-232.

43. Oberbeckmann S, Osborn AM, Duhaime MMB, Carpenter E, Smith K, Colton J, Knapp F, Burns B, Thompson R, Swan S, Moore C, Saal F vom, Cózar A, Echevarría F, González-Gordillo J, Irigoien X, Ubeda B, Hernández-León S, Eriksen M... Yamaji H. 2016. Microbes on a Bottle: Substrate, Season and Geography Influence Community Composition of Microbes Colonizing Marine Plastic Debris. PLoS One 11:e0159289.

44. Erni-Cassola G, Wright RJ, Gibson MI, Christie-Oleza JA. 2020. Early Colonization of Weathered Polyethylene by Distinct Bacteria in Marine Coastal Seawater. Microb Ecol 79:517-526.

45. Dussud C, Hudec C, George M, Fabre P, Higgs P, Bruzaud S, Delort AM, Eyheraguibel B, Meistertzheim AL, Jacquin J, Cheng J, Callac N, Odobel C, Rabouille S, Ghiglione JF. 2018. Colonization of nonbiodegradable and biodegradable plastics by marine microorganisms. Front Microbiol 9:1571.

46. Yoshida S, Hiraga K, Takehana T, Taniguchi I, Yamaji H, Maeda Y, Toyohara K, Miyamoto K, Kimura Y, Oda K. 2016. A bacterium that degrades and assimilates poly(ethylene terephthalate). Science 351:1196-9. 